\title{
Valoración de los conocimientos y prácticas culturales de la agricultura ancestral relacionados a las prácticas agrícolas sostenibles en la comunidad de Nahuizalco
}

\author{
Assessment of the cultural practices and knowledge \\ of ancestral agriculture related to the sustainable agricultural practices \\ in the community of Nahuizalco
}

\section{ISSN 2071-8748 \\ E-ISSN 2218-3345 \\ (c) $\$ 0$ \\ BY NC SA}

Josué Mauricio López-Quintana ORCID: 0000-0003-0888-6574 Universidad Tecnológica de El Salvador

Recibido: 10 de agosto 2019 Aprobado: 29 de noviembre 2019

DOI: https://doi.org/10.5377/entorno.v0i68.8446

URI: http://hdl.handle.net/11298/1126

\section{Resumen}

La agricultura es un proceso de obtención de productos destinados al consumo y comercio, para el bienestar económico y seguridad alimentaria de una población, en este proceso se utilizan una cantidad de recursos a disposición y se invierten cantidades determinadas de mano de obra y materiales. En el caso de Nahuizalco la agricultura representa una forma de vida vinculada a la identidad, la tradición y la religiosidad de sus productores quienes se encargan de producir para generar ingresos a sus núcleos familiares aún conservan un legado ancestral durante las varias etapas y momentos de las siembras. Se realizó un acercamiento etnográfico al proceso de

\section{Abstract}

Agriculture refers to the process of obtaining products for consumption and trade, for the economic wellbeing and food security of a population. In this process, a certain amount of available resources are used and these are invested in a specific amount of labor force and materials. In the case of Nahuizalco, agriculture represents a way of life linked to the identity, tradition and religiosity of its farmers. Those in charge of producing in order to generate income for their families, still retain an ancestral legacy during the different stages and moments of planting the crops. The study included an ethnographic approach to the farming 
cultivo de los cantones que actualmente Organizaciones no gubernamentales se encuentran implementando la visión agroecológica que retoma elementos de los conocimientos ancestrales de las comunidades indígenas de Nahuizalco para el desarrollo sostenible

\section{Palabras clave}

Agricultura - historia - Nahuizalco, El Salvador. Desarrollo agrícola - El Salvador. Cultivo de la tierra - Nahuizalco, EI Salvador. Productividad agrícola.

\section{Introducción}

La temática agrícola y lo relacionado con el trabajo de la tierra y sus productos son un tema que interesa a las autoridades gubernamentales competentes y municipales, como también a pequeños pobladores de una región. De esto se comprende que la agricultura tiene un interés a escala de país, cuyos compromisos están vinculados con la sostenibilidad económica y alimentaria. Asimismo, la empresa privada tiene interés por la producción de cultivos y su procesamiento, para la comercialización de productos utilizados en producir servicios alimenticios.

La agricultura es una actividad entrañablemente humana, producto de miles de años de observación e interacción con el entorno natural; es la manipulación y transformación del ambiente a favor de la necesidad alimentaria humana y de los animales domésticos, actividad consolidada en un grupo humano que posee diversas manifestaciones identitarias. Es una de las actividades humanas que transciende al grado de convertirse en un sistema de creencias, visiones, cosmovisiones y tradiciones antes, durante el tiempo de

La manipulación del ambiente y los elementos relacionados con el contexto natural para la producción de vegetales en busca del bienestar humano puede considerase como un cultivo. Al igual que la manipulación genética de plantas silvestres, cuyo proceso se lleva a cabo para la domesticarlas, es un aspecto que se considera como actividad agrícola que aporta al desarrollo tecnológico y a los cambios culturales (Casas, Caballero, Mapes \& Zárate, 1997). El proceso de consolidación y trasformación de la actividad agrícola, para Villareal y Marín (2008), tiene sus comienzos process in the towns which are currently implementing the agroecological vision that takes up elements from the ancestral wisdom of the indigenous communities of Nahuizalco for sustainable development.

\section{Keywords}

Agriculture - history - Nahuizalco, El Salvador. Agricultural development - El Salvador. Farming- Nahuizalco, El Salvador. Agricultural productivity.

en el movimiento humano hacia ríos, lagos, montañas y las costas, permitiendo zonas de asentamiento en donde era necesario crear patrones de recolección de alimentos vegetales, llevando a los grupos humanos a explorar varios ecosistemas, con esto lograban conocer las propiedades de las plantas y les daban uso para alimentación y otras aplicaciones. Casas y Caballero (1995) mencionan que la agricultura tiene un proceso de transformación del ambiente para crear un contexto artificial que permita al ser humano controlar los recursos a disposición para la producción de una planta.

La región mesoamericana hasido uno de los lugares en donde tuvo lugar la experimentación y un proceso de adaptación y cultivo de plantas para ser domesticadas. A pesar de que se tengan muy pocas evidencias paleobotánicas, este proceso tuvo lugar en todo el continente. Villarreal y Marín (2007) mencionan que en Mesoamérica existió un sistema precerámico que se basó en la recolección de frutos y semillas que aportó al conocimiento para el procesamiento de alimentos que fueron colocados en hornos y fogones para su preparación, lo cual contribuyó al sedentarismo. Estos mecanismos, para el procesamiento, almacenamiento y producción del alimento, en un primer momento debieron de permitir el establecimiento de áreas designadas para tareas domésticas. Pero también existieron otras maneras de transportar estos conocimientos, tal como explica Velázquez (2015) que a lo largo del área mesoamericana hubo diversas rutas de comercio, lo que dio lugar a la apropiación de los territorios y al establecimiento de diferencias, formando la organización y una fuerza económica mediante una economía comercial, ya que no solo se intercambiaba con alimentos. 
El Salvador posee varios lugares donde se encuentra evidencia del pasado agrícola del territorio y del área Mesoamericana, principalmente en Joya de Cerén, que entre sus estructuras y evidencias arqueológicas tiene una milpa con cultivos de maíz y posiblemente de maguey, por lo que Mackee (1995) explica que en el complejo habitacional del sitio se encuentran zonas destinadas a la agricultura, además en senderos alrededor las estructuras estaban organizadas de tal forma que el modo de vida de sus habitantes concordara con las actividades agrícolas. Así mismo menciona Sheets (2007), que en Joya de Cerén se tenía una constante actividad productiva al analizar los surcos de cultivo en diferentes zonas del complejo arqueológico encontrando; también posibles cultivos de yuca. En el sitio existen múltiples linderos que conectan con las estructuras habitacionales, esto puede considerarse como algún tipo de agricultura comunitaria (Dixon, 2013).

Existen otros sitios arqueológicos en el interior del país, como el Cerrón Grande y el Bulevar Monseñor Óscar Arnulfo Romero, y en Chalchuapa, lugares que se encuentran bajo varias capas de ceniza volcánica y que poseen muchos surcos de cultivo. En el caso del bulevar, se tienen evidencias de una milpa (Nobuyuki Ito, Watanabe y Kimura, 2018). En la tefra Cuscatlán se encuentra evidencia de milpas relacionadas con el maíz, debido a esto puede pensarse que el maíz es un cultivo que se encuentra en todos los lugares en donde hay evidencias de actividades agrícolas, con características similares a otros puntos donde también existen evidencias agrícolas que incluso pueden compararse con la forma como se cultiva en la actualidad (Amaroli, 2009).

La tenencia, el trabajo y la producción de la tierra están ligados a los ámbitos políticos, sociales e históricos; pero también poseen una fuerte connotación cultural, un arraigo en la tradición, las creencias, la religiosidad y la identidad que se expresan en los pueblos salvadoreños mediante sus modos de vida. Nahuizalco, municipio del departamento de Sonsonate, es parte de la Reserva de Biosfera de Apaneca-llamatepec, que es un territorio conformado por ecosistemas terrestres o marinos. Certificado por la Unesco para promover el desarrollo sostenible mediante la participación de los sectores locales para la integración de la población con el fin de promover la importancia de la conservación natural y cultural.
En el año 2013, el Ministerio de Medio Ambiente y Recursos Naturales presentó la Estrategia Nacional de Biodiversidad, integrada cuatroestrategiasnacionales: Biodiversidad, Cambio Climático, Recursos Hídricos y Saneamiento Ambiental, como una alternativa para solventar las necesidades medioambientales del país. La primera estrategia aborda la importancia de los conocimientos agrícolas que poseen las comunidades locales, poniendo énfasis en aquellos saberes que poseen acerca de la naturaleza y su diversidad, no solo sobre la agricultura.

Desde la parte cultural y social, la "Monografía indígena del municipio de Nahuizalco", realizada por la Secretaría de Cultura de la Presidencia en el año 2012, presentó la considerable pérdida y degradación ambiental del municipio, al igual que aborda aspectos sociales como la organización de las comunidades por la preservación del agua y la biodiversidad, que está relacionado con las temáticas del arrendamiento de tierras y de la producción agrícola, además de con el pasado histórico y cultural propio de la identidad y las tradiciones que se encuentran en el municipio, entre otras.

En Nahuizalco, actualmente las ONG buscan implementar en las comunidades agrícolas la visión de la agroecología para lograr la sostenibilidad económica, social y ambiental, como en los casos de Sisimitepet, Anal Abajo, Tajcuilujlan y Pushtan, sitios que aún conservan elementos de la identidad indígena; y mucho del conocimiento acerca de la naturaleza se vuelve parte de todo tipo de expresiones culturales que giran en torno a las épocas de siembra y que forman parte de la tradición e historia. De las cuatro comunidades mencionadas proviene un grupo de agricultores, hombres y mujeres, que guardan la tradición y el conocimiento sobre la vida agrícola.

El pasado agrícola de Nahuizalco está documentado a través del registro de los viajes de Pedro Cortés y Larraz al visitar las parroquias del antiguo territorio salvadoreño; menciona que se produce y se cosecha maíz y frijol en gran abundancia. Quien se interesó por la forma de vida y los aspectos agrícolas de Nahuizalco fue el antropólogo sueco Carl Vilhelm Hartman al estudiar las costumbres, tradiciones y creencias de la vida indígena de la zona. El Salvador es el país más pequeño de Centroamérica, pero el más cultivado, 
afirma Hartman, además de comentar que los pobladores de Nahuizalco viven de la tierra, del trabajo que realizan en la producción de cestería y de las huertas, y que siembran variedades del maíz y frijol.

En el trabajo "Reconocimiento Etnográfico de los Aztecas de El Salvador de 1901", Hartman (2001) describe el momento de la siembra de maíz como una expresión cultural; la siembra se realizaba de manera conjunta con sus habitantes, una celebración colectiva que empieza en el momento de dirigirse al lugar donde se realizaba la siembra, esto tenía lugar antes de que la tierra fuese delimitada. Ignacio Barberena (1998) habla sobre que el patrimonio principal de los indígenas es la fabricación de petates y productos derivados del tule, al igual que los cultivos de maíz, frijol, arroz, yuca, azúcar y café.

\section{Método}

Para la estrategia metodológica, se optó por un acercamiento etnográfico a las comunidades agrícolas de Sisimitepet, Anal Abajo, Tajcuilujlan y Pushtan, esto para identificar espacios, contextos, visiones y modos de vida de sus habitantes en relación con todo tipo de expresión cultural relacionada con la agricultura. En Nahuizalco, la tradición, las creencias y la religiosidad de quienes trabajan la tierra se manifiestan en distintos momentos y formas durante el proceso de preparación y producción agrícola, volviéndose parte de la identidad de sus habitantes, especialmente de aquellos que poseen una herencia ancestral y practican los conocimientos transmitidos por sus padres y abuelos.

\section{Fasese de la investigacion en}

Nahuizalco

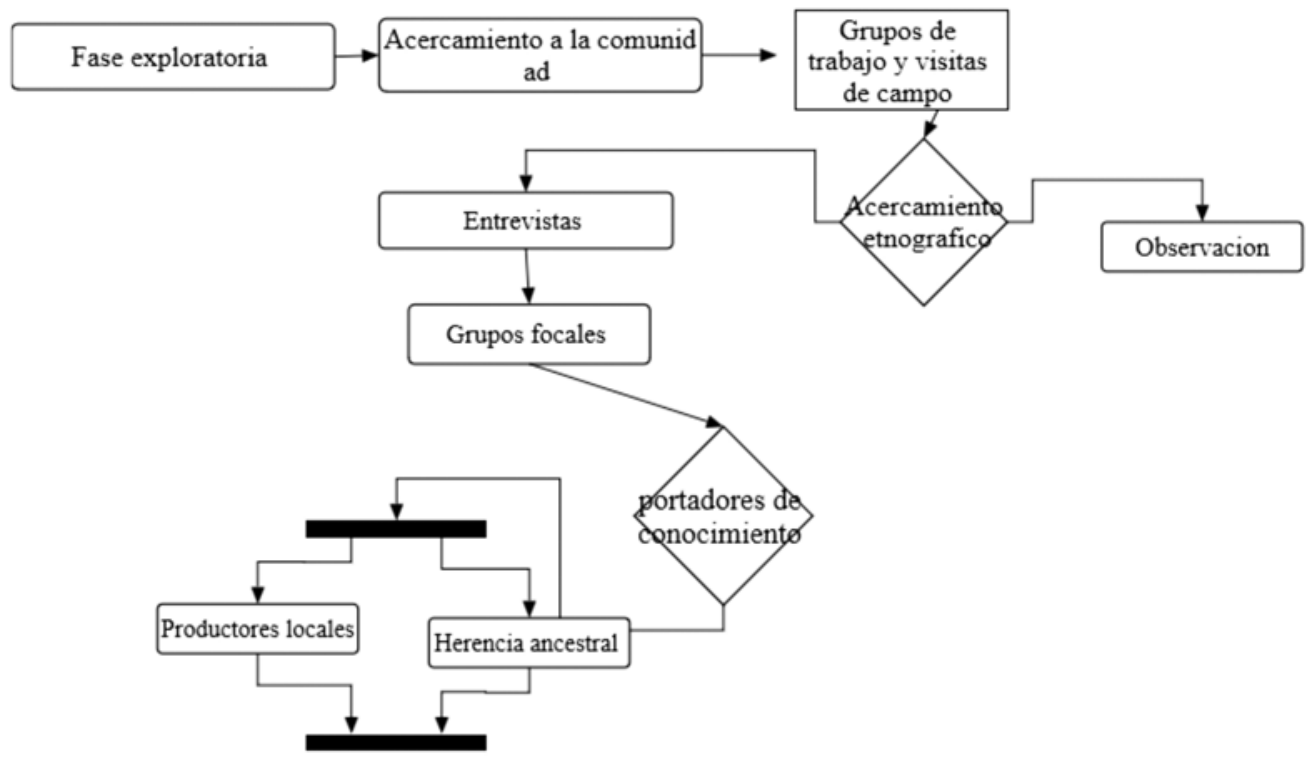

Fase exploratoria: se realizó un acercamiento a la comunidad mediante la coordinación de la Fundación para el Desarrollo Socioeconómico y Restauración Ambiental (Fundesyram), que tiene cierto conocimiento acerca de la producción agrícola local, al igual que ha logrado identificar ciertas prácticas y saberes sobre la manipulación de ciertos cultivos representativos de la comunidad local. Acercamiento etnográfico: en el caso de Nahuizalco, para visualizar el proceso de implementación de la visión agroecológica, así como también para la parte de identidad, simbolismo y tradiciones agrícolas, se usó del método etnográfico, tal como mencionan Quezada y Quintana, (2015), que la implementación etnográfica se vuelve una herramienta precisa que permite indagar en los escenarios socioculturales que tienen lugar en las comunidades. Además del método etnográfico, la investigación se realizó desde la perspectiva emic. Como menciona Castillejo (2013), aquellas expresiones de la cultura que son parte de la identidad de un pueblo van a ser comprendidas desde la misma comunidad, al igual que los conceptos, términos y significados, por tanto, las expresiones y conocimientos agrícolas son analizadas desde el marco cultural local. 
Se realizaron visitas a las parcelas para observar el proceso de cultivo; también se entrevistó a miembros del Comité de Portadores de Conocimiento Ancestral de Nahuizalco, entre ellos hombres y mujeres experimentados en labores agrícolas y que se han dedicado a dicha forma de vida desde una temprana edad, cuya característica principal son parte de las familias que poseen rasgos de la identidad y cosmovisión indígena, y manifiestan algunos de esos elementos a través de diversas expresiones en la agricultura Para el análisis de datos, se utilizó NVivo como software para interpretación de datos cualitativos, generando esquemas de relación por conglomerados. Además, los relatos de experiencias y los conocimientos compartidos y documentados permiten visualizar el comportamiento cultural de la comunidad.

\section{Resultados}

En los cantones de Nahuizalco Sisimitepet, Pushtan, Tajcuilujlan y Anal Abajo las familias conservan ciertos conocimientos acerca de la producción, cuido y manejo de cultivos, una clase de saberes que han logrado transmitirse por medio de la oralidad y la práctica. Tratándose no solo de la producción y distribución entre la comunidad, o bien sea para la comercialización, la agricultura tiene un lugar importante en la identidad ancestral. Algunas de sus familias continúan replicando ese legado a través del ciclo agrícola, las temporadas de preparación de la tierra. Los cuidados y las formas en que se va a tratar el cultivo y su cosecha son una parte del conocimiento adquirido de la interacción del ser humano y su medio. La agricultura en los cantones es una manera en que algunas familias subsisten, ya sea consumiendo o comerciando sus cosechas; de esa forma pueden percibir ingresos. Asimismo, en la agricultura se transmiten aquellos elementos de la identidad $y$ cosmovisión que son replicados en diversos tipos de momentos; expresiones culturales que toman lugar en la comunidad. El tratamiento de la tierra, los conocimientos sobre plagas, tiempos de siembra, abono y el tiempo de cosecha son aspectos que están determinados por los mismos elementos naturales que contribuyen a medir los tiempos y a realizar los deberes correspondientes al trabajo de la tierra, así como intervenir en otros más de carácter cultural.

Movimientos lunares

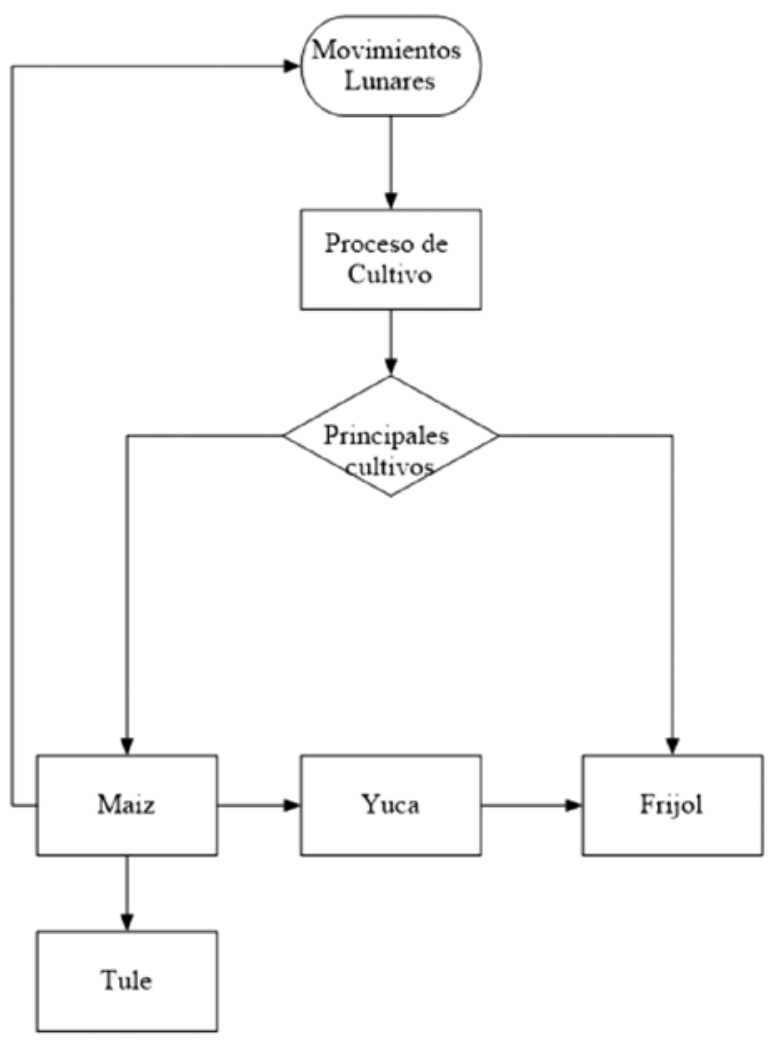


La luna tiene una importante representación simbólica como elemento que está presente en los momentos de la vida social de sus habitantes, quienes poseen ese legado de generación en generación y que algunos de sus más ancianos aun toman en cuenta. Se trata de un sistema ancestral que se basa en la interpretación de las fases la luna, teniendo un importante significado cosmológico para las comunidades indígenas de Nahuizalco e Izalco, retomando esas fases para realizar otras actividades de la vida cotidiana. Schultze Jena (1997) explica que ciertos aspectos relacionados a la luna la parte agrícola, en las que se distinguen tres aspectos importantes para cultivar: los conocimientos que son empleados para llevar los tiempos de peina (preparación de la tierra), la siembra y la dobla (cosecha). Como sucede en las siguientes fases lunares, en la luna nueva se evita sembrar por la creencia de que lo que se siembre no echara raíces, al igual que sembrar en la luna en cuarto menguante, mientras que la en la luna tierna es el momento idóneo para sembrar.

De esa forma lo explica doña Teodora (2018), del cantón Sisimitepet:

No se puede sembrar. Solo, por ejemplo, nosotros tenemos la costumbre de sembrar cuando es 11 de luna. Por ejemplo, ahorita va a ser 11 (once de luna) el 24 de julio. Ese día vamos a sembrar unos palitos todavía, porque sabemos que no se muera, porque creemos que Dios nos va a enviar la lluvia, y va a llover, y no se van a morir los palitos. Así que vamos a sembrar el 24 de julio; ese es el día perfecto. Otros siembran el 29 de luna, un día que se vaya la luna. Otros siembran en creciente. La yuca se puede sembrar en creciente. Para que crezca, la yuca se puede sembrarse en creciente; cuatro o cinco días de luna.

Cultivos de yuca, frijol y maíz en los cantones de Sisimitepet, Pushtan, Tajcuilujlan y Anal Abajo de Nahuizalco

\section{La siembra de yuca}

Se inicia consiguiendo los vástagos de la yuca, se cortan y se colocan llevando una posición; pero antes de ser sembrados, según la tradición, el sembrador tiene que frotarse las manos con la planta matali (Tradescantia zebrina) y después acariciar el vástago e irlo sembrando a $40 \mathrm{~cm}$ entre cada estaca. El sembrador debe de coincidir con las fases de luna. En este caso se trata de 11 de luna. La persona que siembra, abre la tierra para enterrar los vástagos de yuca, explicando que si la lluvia viene del sur los vástagos deben de ir en una posición que no les caiga directamente la lluvia. La estaca (yuca) debe quedar completamente enterrada. "(...) Por medio de ustedes, pues, verdad, y por medio de esta plantita vamos a sembrar esta plantita. Espero que Dios nos dé fuerza. Primero a ustedes, en primer lugar, ya que Dios nos tiene en vida, ¿verdad? Y así, que esta yuquita salga bien (...)". Habiendo dicho esas palabras, el agricultor, en este caso uno de los hijos de don Margarito, frota las raíces de la planta matali; y con sus manos impregnadas de la tierra que tiene dicha planta frotan las estacas de yuca y comienzan a enterrarlas. En este caso fueron sembradas de seis a ocho estacas; y aproximadamente en cuatro meses se verán las cosechas de yuca. Uno de los agricultores menciona que el 3 de mayo ("Día de la Cruz") el párroco llega a la comunidad y todos los agricultores llevan sus semillas, y él hace la bendición y la oración de las cinco velas de cinco colores: amarillo, azul, negro, etc., y cada una está puesta en un círculo como una estrella.

De esa forma se siembra la yuca. Esperando haber cumplido con la tradición del frotamiento y habiendo enterrado la yuca, el grupo regresa a la plaza de Nahuizalco para continuar en el mercado. Debe destacarse que el sistema lunar juega un papel importante para el cultivo local. Los agricultores concuerdan en sus fechas de siembra, según las fases de la luna, el día de la siembra de la yuca. Las fases lunares son importantes para la agricultura, pero no solo para este aspecto, sino que también para la vida cotidiana. Esto forma parte de las creencias locales. Por ejemplo, en el ámbito reproductivo o en salud, ya que el joven comenta que no es lo mismo herirse en un día que la luna no esta creciente a que cuando está llena. La figura de la luna tiene una connotación femenina, mientras que el Sol es considerado como el "abuelo sol", con una entidad masculina.

\section{El cultivo de frijol}

La siembra del frijol se llevó a cabo en el cantón Anal Abajo actividad correspondiente al calendario lunar (luna llena) y de acuerdo con el cambio de cultivo conveniente, ya que estos se rotan bajo la creencia de que la tierra necesita descansar de ciertos cultivos, para que estos den buenas cosechas. Debido a que para el campesino la tierra representa un ser vivo que le proporciona el alimento, es por eso que llega un momento cuando se cansa de generar 
los mismos cultivos. El agricultor utiliza un lazo para poder trazar la línea en donde serán sembradas las semillas, se toma el chuzo (tronco de madera con punta) para poder hacer agujeros en la tierra; y se toma otro madero para golpearlo, para que la fuerza permita que el chuzo sea enterrado en el suelo, mientras se hacen los agujeros se van colocando tres semillas en cada uno. Del otro lado, otra persona coloca ceniza conforme a cada surco, siguiendo la línea trazada; y así se hace en cada surco.

En la siembra, se observó cómo se transmiten los conocimientos y se aprenden las habilidades para con la nueva generación, por lo que se tuvo una participación importante durante esta. En esta ocasión, niños de la comunidad fueron a aprender a sembrar. En la actividad de la siembra, el agricultor enseña poniendo su ejemplo primero: toma el chuzo, lo golpea con otro madero y hace el agujero, luego da dos pasos para hacer el siguiente. Esto lo hace dos veces y luego pone al joven a hacerlo, mientras el otro agricultor comienza a colocar ceniza en las líneas ya trazadas, en las que se ha depositado semilla de frijol.

\section{La siembra de maíz}

El maíz es uno de los cultivos primordiales en la producción agrícola de Nahuizalco; es uno de los cultivos representativos de la identidad indígena y del diario vivir de los agricultores. Nunca falta la producción de maíz en la milpa, al igual que el frijol, se cultiva de manera continua para el sustento diario. El maíz tiene una connotación simbólica en la cosmovisión de la comunidad indígena, tal arraigo cultural tiene que actualmente tanto la siembra como la cosecha representan un momento de alegría y de convivencia entre la comunidad. Es una visión que permanece en las actuales comunidades que poseen rasgos de identidad indígena y, asimismo, que se transmite en la familia y de generación en generación, mientras que otros agricultores manifiestan ciertos elementos que se realizan en la siembra del maíz, pero que se llevan a cabo por tradición. La siembra del maíz tiene elementos ceremoniales y tradicionales que son visibles durante todo su proceso, desde que se va a cultivar hasta la cosecha, en donde la comunidad y las familias participan.

Hartman (2000) describe la variedad de productos comestibles derivados del maíz, al igual que la producción de sus huertas, muy ricas en vegetales y especias. Existe otro elemento en la descripción de este investigador. Se trata del chuzo, un objeto importante para las siembras del frijol y el maíz, además es un interesante elemento simbólico. Hartman menciona que estos objetos tienen la función de proteger los cultivos de los huracanes. Pero los chuzos tienen otra función, también importante, pues es una herramienta para depositar la semilla y el chilate, afirman los agricultores y practicantes; es una manera de mostrarle a la tierra que el agricultor se interesa por su bienestar, y el depositarle chilate es una muestra de agradecimiento por ocupar el espacio y sus nutrientes para producir alimentos; se vuelve una cortesía, un respeto para con el espacio simbólico que es la milpa. En la actualidad persisten algunos elementos como el chuzo, la preparación del chilate, el refrigerio y el almuerzo para los mozos; al igual que en las doblas, la preparación de tamales y chilate para repartir.

Así lo expresa la 'niña' Élida Hernández (2018) del cantón Tajcuilujlan:

Ella... Bueno, cuando ella dice que iba a sembrar, ella estaba temprano. Hacía chilate, llevaba dulce, iba a orar; en el guacal iba a enflorar a las cuatro esquinas. En la siembra, le pedía a Dios. Y luego en medio del terreno hacía el hoyo y ahí enterraba el chilate; y lo enterraba en las cuatro esquinas... Lo enterraba. De ahí echaba su agua bendita -que le dicen-y lo rociaba, y ahí se quedaba. Y hacían una cruz de palo de santo y ahí la dejaban en la siembra. Luego decía ella "Por favor — les decía a los mozos-, yo les voy a llamar que vayan a almorzar, pero no me vayan a dejar los chuzos aventados. "Por qué" — decían ellos-. "Porque, si no, va a venir la tormenta; me lo va a votar todo en la milpa. Porque el chuzo tiene que quedar sembrado". A todos los mozos les decía así. Y ya, cuando había el elote, a todos les repartía. A los vecinos se ponía repartir. Cuando era la dobla, le daba su poquito a cada quien. Hacía tamales, hacía tuyuyo. Todo lo daba a repartir; pero más la bendecía Dios. Pero no era tacaña. Ella a todo mundo le regalaba... Ella regalaba. Y gracias, bien dicho, que todo el que es amable con la persona dondequiera hay amistad, tengamos dinero o no tengamos, pero siempre comemos 


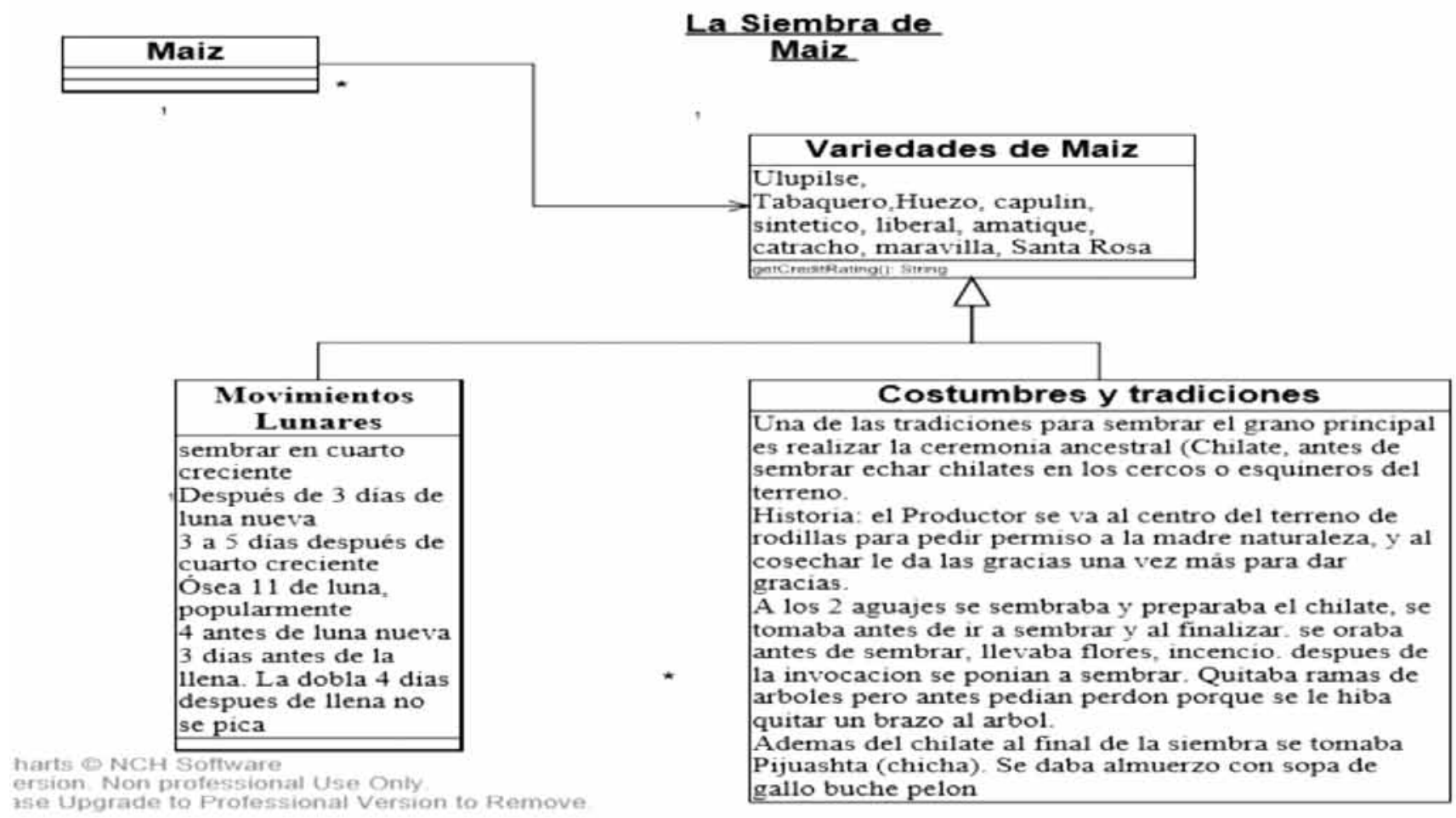

\section{Nodos conglomerados por similitud de codificación}

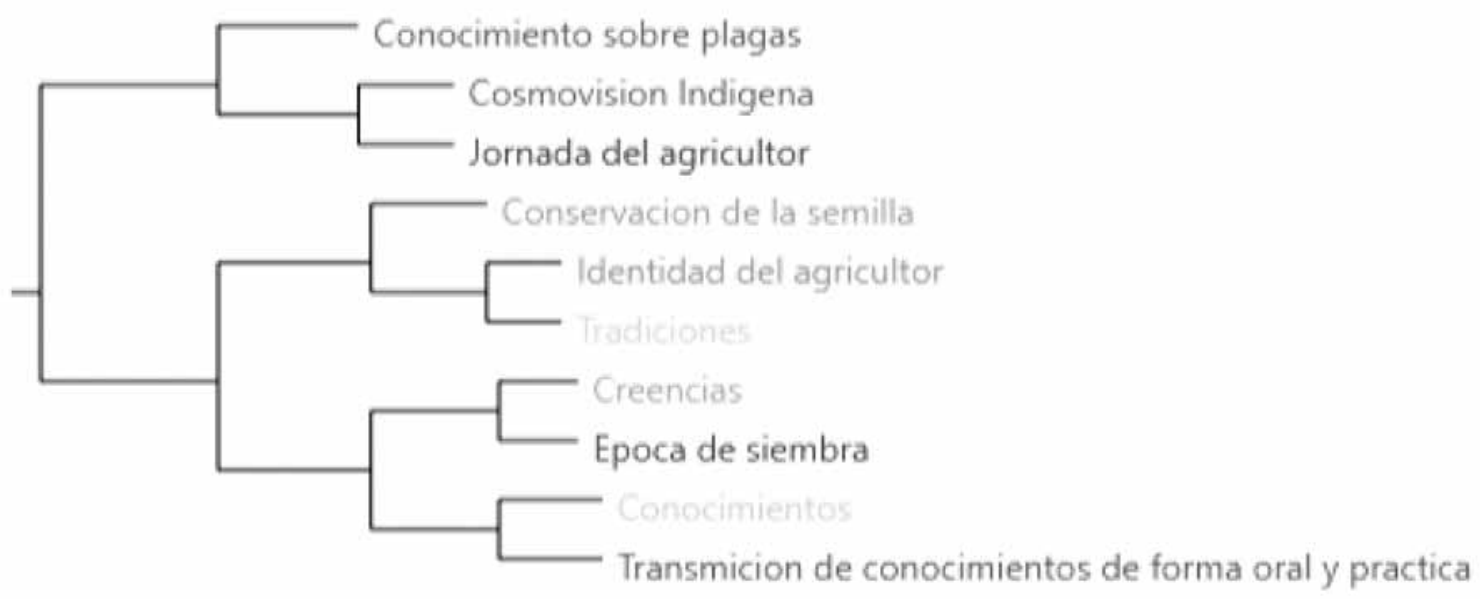

La transmisión de conocimientos de forma oral y práctica está totalmente vinculada con los conocimientos, que incluyen el manejo de recursos naturales como el suelo, los abonos, el agua y la semilla misma. Se trata de una dinámica cultural, porque el conocimiento que se encuentra en la parte intangible se transmite de manera oral a las nuevas generaciones y se lleva a la práctica. Ahora las creencias se manifiestan efectivamente durante la época de la siembra, para la protección y desarrollo de los cultivos. Las tradiciones están emparentadas con la identidad del agricultor; son una parte fundamental en la realización de las tradiciones, como la celebración de la bendición de 
la semilla, la Semana Santa, el "Día de los Difuntos" y los Canchules etc. Algunas de estas celebraciones se llevan a cabo en otras partes del país, pero en Nahuizalco tienen un notable arraigo cultural en la población.

La conservación de la semilla es un elemento vinculado con la tradición. Mediante el acercamiento etnográfico se identificó que el agricultor, por tradición, recibe su herencia material representada en las semillas; de esa manera puede continuar realizando el proceso agrícola, permitiéndole asegurar la alimentación de su familia y seguir practicando el conjunto de costumbres y creencias, las cuales forman parte de su identidad como agricultor; y en el caso del agricultor indígena, de su cosmovisión.

\section{Nodos conglomerados por simillitud de palabra}

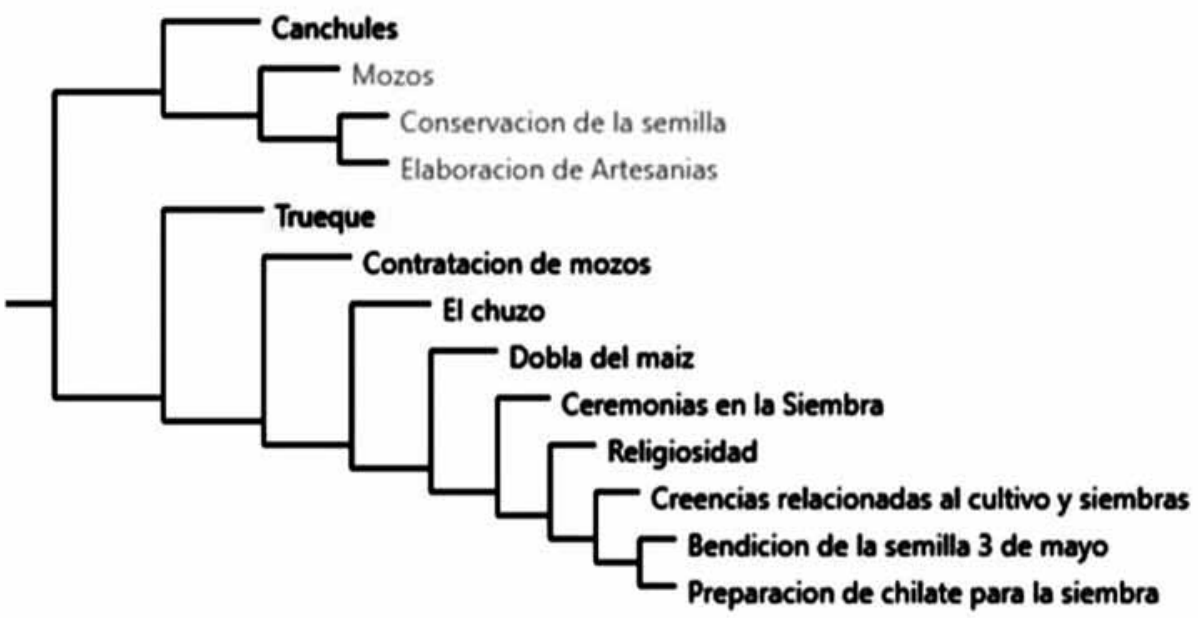

La preparación del chilate para consumirlo durante la siembra es un elemento que tiene una relación directa con el pensamiento colectivo agrícola, debido a que la comunidad indígena realiza una actividad para llamar a sus familias y conocidos, para efectuar la bendición de sus semillas guardadas de la anterior cosecha o de anteriores. En esta actividad, se pide a Dios y a los elementos de la naturaleza que propicien buenas cosechas y que tengan protección de los huracanes. Esto a su vez se relaciona con las creencias acerca de la preparación, cuidados y relación con la milpa, la naturaleza y los cultivos, aspectos que permanecen en la identidad de los pobladores, quienes procuran cumplir con las enseñanzas transmitidas de sus abuelos.

La religiosidad tiene un papel importante en el proceso agrícola, en la preparación (peina), siembra y la cosecha (dobla); se reza un padrenuestro, ya sea para pedir por la seguridad de la milpa o para agradecer la productividad.
Esto conlleva a otro aspecto, que son las ceremonias en las siembras, que tienen que ver con la identidad indígena, que actualmente en cantones como Sisimitepet han dejado de realizarse debido a que los abuelos, figuras importantes para la comunidad, han comenzado a morir, y otros padecen de enfermedades que no les permiten movilizarse. Otro elemento que sobresale son las doblas (se llama así a la corta del maíz o de otro cultivo), que es una parte del cultivo con varios aspectos culturales. Para esta actividad, se prepara chilate y se hacen tamales para repartir; es una celebración que se hace en agradecimiento por la cosecha, por ello se comparte entre la comunidad.

Otros aspectos que son parte de la siembra del maíz son el chuzo y la contratación de mozos. El chuzo es el objeto con el que se abre la tierra para depositar la semilla es la herramienta con la que el ser humano, en este caso el agricultor, protege sus cultivos y también deposita la semilla para darle vida a su milpa. De esta afirmación, se 
comprende que es la mano del agricultor la que mueve el instrumento para asegurar la vida de la comunidad y para darle continuidad a los conocimientos que ha heredado de sus padres. Los mozos son los ayudantes que se encargan de apoyar en la labor agrícola al momento de la peina, la siembra y la dobla; ellos acompañan todo el proceso. Por eso puede comprenderse la relación con el chuzo, ya que el mozo es quien realiza la tarea de abrir la tierra con dicho objeto.

El trueque $o$ intercambio de productos es un aspecto que se mantiene en la comunidad, en la población de adultos mayores, como parte de las tradiciones y costumbres que permiten la preservación de la semilla y la diversidad de cultivos, en el caso de los maíces y frijoles. La conservación de la semilla y elaboración de bancos de semillas, permite asegurar los materiales genéticos e insumos, como por ejemplo el tule, para elaborar los productos artesanales como los petates y las canastas que se comercializan en el mercado municipal de Nahuizalco

\section{Conclusiones}

Un elemento representativo de las expresiones culturales agrícolas es la siembra del maíz; desde sus primeras etapas se muestran características relacionadas con las creencias, las tradiciones y la cosmovisión, tratándose de uno de los aspectos que permite la conservación de la de la identidad. La siembra del maíz está determinada por el calendario lunar; el astro y sus movimientos inciden en la disposición y acción del agricultor para sembrar.

Se comprende que la agricultura es una de las principales actividades económicas del municipio de Nahuizalco. Dicha actividad permite la subsistencia familiar de las poblaciones en los sectores rurales. En Nahuizalco existe una dinámica socioeconómica que combina elementos de la identidad, tradiciones y costumbres, que se ven reflejados en la vida agrícola. Las familias agricultoras se preparan para los periodos de siembra, desde mayo a septiembre; y en parte de los últimos meses de octubre y noviembre se están haciendo las últimas cosechas y doblas. Nahuizalco es uno de los ejemplos de productividad agrícola que a través de la historia ha mantenido dicha actividad; y uno de los lugares en donde se está volviendo a implementar las raíces de la agricultura ancestral, al incentivar a la población a recuperar y revalorar los conocimientos que poseen los pobladores de los cantones de Sisimitepet, Pushtan, Tajcuilujlan y Anal Abajo de Nahuizalco.
El maíz tiene una connotación simbólica en la cosmovisión de la comunidad indígena, de tal arraigo cultural que se consideran actualmente, tanto la siembra como la cosecha, como momentos de alegría y de convivencia para la comunidad. Es una visión que permanece en las actuales comunidades que tienen rasgos de identidad indígenas, y, asimismo, que se transmiten en familia de generación en generación La siembra del maíz tiene elementos ceremoniales y tradicionales que son visibles durante todo su proceso desde que se va a cultivar hasta la cosecha. Probablemente en algún momento de la historia de Nahuizalco fue una festividad de la comunidad indígena, mientras que hoy en día se practica por respeto al legado ancestral.

\section{Referencias}

Amaroli, P. y Dull, R. (2009). Milpas prehispánicas en El Salvador [versión de Adobe Acrobat Reader]. Recuperado de http://www.fundar.org.sv/referencias/ milpas.pdf

Casas, A. y Caballero, J. (octubre-diciembre, 1995). Domesticación de las plantas y origen de la agricultura en Mesoamérica. Ciencias, 40, 36-45. Recuperado de https://www.revistaciencias.unam.mx/es/146-revistas/ revista-ciencias-40/1196-domesticación-de-plantas-yorigen-de-la-agricultura-en-mesoamérica.html

Casas, A., Caballero, J., Mapes, C. y Zarate, S. (1997). Manejo de vegetación, domesticación de plantas y origen de la agricultura en Mesoamérica. Sociedad botánica de México, (61), 31-47. doi: https://doi.org/10.17129/ botsci. 1537

Corona Velázquez, D. A. (2015). La expansión territorial económica en Mesoamérica y Mesoamérica septentrional por medio del intercambio. Geografia Ensino \& Pesquisa, (19), 59-68. doi: 10.5902/22364994/19346

Cortés y Larraz, P. (2000). Descripción geográfico moral de la Diócesis de Goathemala. San Salvador, El Salvador: Dirección de publicaciones e Impresos.

Dixon, C. C. (2013). Agricultura de yuca. En P. Sheets (Ed.), Joya de Cerén patrimonio cultural de la humanidad 19932013. (pp. 251-252). San Salvador, El Salvador: Editorial Universitaria. Recuperado de https://www.colorado. edu/anthropology/si tes/default/files/attached-files/ joya_sheets_23_oct_2013.pdf

Fundación para el Desarrollo Socioeconómico y Restauración Ambiental. (2017). Estudio Agro socio económico participativo de 4 comunidades de los cantones 
Pusthan, Tajcuilujlan, Sisimitepet y Casamuluco [versión de Adobe Acrobat Reader]. Recuperado de www.fundesyram.info/document/PDFPUB/ Sistematizacion. pdf

Hartman, C. V. (junio, 2001). Reconocimiento etnográfico de Ios Aztecas en El Salvador. Mesoamérica, 22(41), 146191. Recuperado de https://dialnet.unirioja.es/servlet/ arti culo? codigo $=2453379$

Ito, N. Watanabe, T. y Kimura, M. (2018). Reconstrucción de la agricultura prehispánica en El Salvador previo a la erupción volcánica, a través del análisis de suelos. Kóot, 9, 26-36. doi: https://doi.org/10.5377/koot. v0i9.5903

Mckee, B. (1997). La reutilización de materiales arqueológicos el sitio Céren, El Salvador [versión de Adobe Acrobat Reader]. Recuperado de http://www.asociaciontikal. com/wp-content/uploads/2016/11/08.94_-_ McKee. pdf
Romero Quesada, M. A. y Hernández Quintana, A. R. (2015). El método etnográfico y su relación con el análisis de dominio. Biblios, 61, 70-84. Recuperado de https:// www.redalyc.org/articulo.oa?id=16144489005

Schultze, J. L., Menjivar Rieken, G. y Parada Fortin, A. (1977). Mitos y leyendas de los Pipiles de Izalco. Santa Ana, El Salvador: Ediciones Cuscatlán.

Sheets, P. (2011). Resumen de investigaciones geofísicas y arqueológicas al sur de Joya de Cerén, 2007. La universidad, 15, 208-2014. Recuperado de http:// revistas.ues. edu.sv/index.php/launiversidad/article/ view/191/241

Zizumbo Villareal, D. y García Marín, P. C. (julio-diciembre, 2008). El origen de la agricultura, la domesticación de plantas y el establecimiento de corredores biológicoculturales en Mesoamérica. Geografía Agrícola, (41), 85-113. Recuperado de https://www.redalyc.org/ pdf/757/75711472007.pdf 